\title{
Whole brain optoacoustic tomography reveals strain-specific regional beta-amyloid densities in Alzheimer`s disease amyloidosis models
}

Ruiqing $\mathrm{Ni}^{1,2^{*}}$, Xose Luis Dean-Ben ${ }^{1,3^{*}}$, Daniel Kirschenbaum ${ }^{4}$, Markus Rudin ${ }^{1}$, Zhenyue Chen $^{1,3}$, Alessandro Crimi ${ }^{4}$, Fabian F. Voigt ${ }^{2,5}$, K. Peter R. Nilsson ${ }^{6}$, Fritjof Helmchen ${ }^{2,5}$, Roger Nitsch $^{7}$, Adriano Aguzzi ${ }^{4}$, Daniel Razansky ${ }^{1,2,3^{*}}$, Jan Klohs $^{1,2 *}$

${ }^{1}$ Institute for Biomedical Engineering, ETH \& University of Zurich, Zurich, Switzerland

${ }^{2}$ Zurich Neuroscience Center (ZNZ), Zurich, Switzerland

${ }^{3}$ Faculty of Medicine and Institute of Pharmacology and Toxicology, University of Zurich,

Switzerland

${ }^{4}$ Neuropathology, Universitätsspital Zurich, Switzerland

${ }^{5}$ Brain Research institute, University of Zurich, Switzerland

${ }^{6}$ Division of Chemistry, Department of Physics, Chemistry and Biology, Linköping University, Linköping, Sweden

${ }^{7}$ Institute for Regenerative Medicine, University of Zurich, Switzerland

*Contributed equally

Correspondence to:

Jan Klohs

Institute for Biomedical Engineering, University of Zurich \& ETH Zurich

Vladimir-Prelog-Weg 4, 8093 Zurich, Switzerland

Tel.+41 446337629;

Fax.+41 446331187

Email: klohs@biomed.ee.ethz.ch 


\begin{abstract}
Deposition of beta-amyloid $(A \beta)$ deposits is one major histopathological hallmark of Alzheimer's disease (AD). Here, we introduce volumetric multi-spectral optoacoustic tomography (vMSOT), which covers $10 \times 10 \times 10 \mathrm{~mm}^{3}$ field-of-view, capable of 3D whole mouse brain imaging. We show for the first time the optoacoustic properties of oxazine-derivative AOI987 probe, which binds to $\mathrm{A} \beta$, and the application of vMSOT for the quantification of brainwide $\mathrm{A} \beta$ deposition. Administration of AOI987 to two common transgenic mouse strains of $\mathrm{AD}$ amyloidosis led to a retention of the probe in A $\beta$-laden brain regions. Co-registered of vMSOT data to a brain atlas revealed strain-specific pattern of AOI987 uptake. A comparison with ex vivo light-sheet microscopy in cleared mouse brains showed a good correspondence in $A \beta$ distribution. Lastly, we demonstrate the specificity of the AOI987 probe by immunohistochemistry. vMSOT with AOI987 facilitates preclinical brain region-specific studies of $A \beta$ spread and accumulation, and the monitoring of putative treatments targeting $A \beta$.
\end{abstract}

Key words: Alzheimer's disease; amyloid-beta; animal model; light-sheet microscopy; optoacoustic tomography; 


\section{Introduction}

Alzheimer's disease (AD) is the most common type of dementia ${ }^{1,2}$. The abnormal acculumation and spread of amyloid- $\beta$ (A $\beta$ ) deposits play a central role in the pathogenesis of $A D$ and leads to downstream pathophysiological events ${ }^{3,4}$. A $\beta$ oligomers, diffuse and fibrillar plaques, can impair neuronal and synaptic function (e.g., long-term potentiation) and selective neuronal loss, and thus contribute collectively to the symptomatology of $\mathrm{AD}^{5}$. Positron emission tomography (PET) imaging of aberrant $\mathrm{A} \beta$ deposits with amyloid probes ${ }^{11} \mathrm{C}$-PIB ${ }^{6},{ }^{18} \mathrm{~F}$-flutemetamol ${ }^{7},{ }^{18} \mathrm{~F}-$ florbetapir ${ }^{8},{ }^{18}$ F-fluobetaben ${ }^{9,10}$ has been established as diagnostic pathological biomarker for AD pathology in the clinical setting and was included as a new diagnostic criterion ${ }^{11}$. Higher cortical $A \beta$ loads were reported in the brain from patients with mild cognitive impairment and AD compared to healthy controls using amyloid PET imaging ${ }^{6,8,9,10}$. PET studies have provided evidence that the accumulation of $\mathrm{A} \beta$ deposits are early and initiating events in the pathogenesis of $\mathrm{AD}^{12}$.

A $\beta$ pathology has been successfully modelled in transgenic engineered mouse lines of cerebral amyloidosis, that have proved useful for translational research ${ }^{5}$. A $\beta$ plaque appearance and spread have been monitored postmortem with histopathology, and resemble in same strains the patterns typically seen in AD patients ${ }^{2,13,14}$. Nevertheless, procedures require extensive lengthy tissue processing and can only be performed cross-sectionally at termination of the experiment. In vivo two-photon and optoacoustic microscopic imaging approaches using PIB, methoxy-X04 $15,16,17$, temperature sensitive agents ${ }^{18}$, Congo red ${ }^{19}$, and HS-169 ${ }^{20}$ have enabled monitoring of $\mathrm{A} \beta$ growth and disease-modifying therapeutic strategies at the microscopic scale with very high resolution. However, these methods are generally invasive and suffer from a limited field-of- 
view (FOV) and/or low penetration depth, where $A \beta$ accumulation can only be observed in a small brain region.

Similar to humans, PET imaging of $\mathrm{A} \beta$ across the whole brain has been performed in mice using ${ }^{11} \mathrm{C}-\mathrm{PIB}{ }^{21},{ }^{11} \mathrm{C}-\mathrm{AZD} 2184{ }^{22},{ }^{18}$ F-fluobetaben ${ }^{23},{ }^{18}$ F-florbetapir ${ }^{24}$ and labeled antibodies ${ }^{25}$. While it is a sensitive and quantitative imaging modality, the limited resolution $(\sim 1 \mathrm{~mm})$ relative to the mouse brain dimensions $\left(\sim 10 \times 8 \mathrm{~mm}^{2}\right)$ hamper to monitor the spread and accumulation of $\mathrm{A} \beta$ in smaller brain regions; and the low availability of radiotracer facilities restrict its usage for widespread mouse brain imaging. Optical imaging modalities such as near-infrared fluorescence (NIRF) using amyloid probes AOI987 ${ }^{26,27}$, or CRANAD-2 ${ }^{28}$ and fluorescent molecular tomography (FMT) using AOI987 ${ }^{27}$ have been shown to provide an alternative for small animal A $\beta$ imaging, but these methods are affected by light scattering and absorption, which limit the achievable spatial resolution and quantification accuracy. Therefore, non-invasive 3D imaging of $\mathrm{A} \beta$ deposition across the whole mouse brain with high sensitivity and high spatial resolution has so far not been possible.

Multi-spectral optoacoustic tomography (MSOT) is rapidly evolving to become an established non-invasive imaging modality in preclinical studies involving mice models of many human diseases 29,30,31. Volumetric MSOT (vMSOT) further enables mapping the bio-distribution of spectrally distinctive contrast agents in entire three-dimensional (3D) regions at multiple spatial and temporal scales ${ }^{29,30,31}$. In contrast to conventional optical imaging, the spatial resolution of vMSOT is not governed by photon scattering but by ultrasound diffraction, thus it can achieve a much higher spatial resolution. As opposed to optoacoustic (OA) microscopy, vMSOT enables high-resolution mapping of endogenous tissue chromophores or exogenous probes at millimeter 
to centimeter scale depths $32,33,34$. We recently, developed a high-resolution vMSOT systems, which enabled efficient real-time volumetric imaging of the entire mouse brain with spatiotemporal resolution and penetration depth far beyond the limits of optical microscopes ${ }^{32,35}$. Herein, we demonstrate the unique capabilities of vMSOT assisted with a NIR oxazine derivative $\mathrm{A} \beta$ probe $\mathrm{AOI} 987^{26}$ for in vivo whole brain mapping of $\mathrm{A} \beta$ deposits in two common mouse models of AD cerebral amyloidosis ${ }^{36}$.

\section{Materials and methods}

\section{Animal models}

Six transgenic $\operatorname{arc} \mathrm{A} \beta$ mice overexpressing the human APP695 transgene containing the Swedish (K670N/M671L) and Arctic (E693G) mutations under the control of prion protein promoter and five age-matched non-transgenic littermates of both sexes were used in this study (18-24 monthsof-age) ${ }^{36,37}$. ArcA $\beta$ mice are characterized by a pronounced amyloid deposition, cerebral amyloid angiopathy and vascular dysfunction ${ }^{14,38,39,40}$. Three APP/PS1 mice ${ }^{41}$ overexpressing the human APP695 transgene containing K670N/M671L and PS1 mutations under the control of prion protein promoter and three age-matched non-transgenic littermates of both sexes (16 months-of-age) were also imaged. Animals were housed in ventilated cages inside a temperaturecontrolled room and under a 12-hour dark/light cycle. Pelleted food (3437PXL15, CARGILL) and water were provided ad-libitum. All experiments were performed in accordance with the Swiss Federal Act on Animal Protection and were approved by the Cantonal Veterinary Office Zurich (permit number: ZH082/18). All procedures fulfilled the ARRIVE guidelines on reporting animal experiments. 


\section{vMSOT system}

The vMSOT set up for in vivo mouse brain imaging is illustrated in Fig. 1a. It is a further development of functional optoacoustic neuro-tomography (FONT) approach ${ }^{32,35}$. Briefly, a short-pulsed (<10 ns) laser was used to provide an approximately uniform illumination profile on the mouse brain surface with optical fluence $<20 \mathrm{~mJ} / \mathrm{cm}^{2}$. The excited OA responses were collected with a custom-made spherical array (Imasonic SaS, Voray, France) of 512 ultrasound detection elements with $7 \mathrm{MHz}$ central frequency and $>80 \%$ bandwidth. The array features a central aperture with $8 \mathrm{~mm}$ diameter and 3 lateral apertures with $4 \mathrm{~mm}$ diameter located at $45^{\circ}$ elevation angle and equally spaced $\left(120^{\circ}\right)$ in the azimuthal direction. A custom-made optical fiber bundle (Ceramoptec GmbH, Bonn, Germany) with 4 outputs was used for guiding the laser beam through the apertures of the array. The optoacoustic signals were digitized at 40 megasamples per second with a custom-made data acquisition system (DAQ, Falkenstein Mikrosysteme GmbH, Taufkirchen, Germany) triggered with the Q-switch output of the laser.

\section{vMSOT of the mouse brains}

Mice were anesthetized with an initial dose of $4 \%$ isoflurane (Abbott, Cham, Switzerland) in an oxygen/air mixture $(200 / 800 \mathrm{~mL} / \mathrm{min})$, and subsequently maintained at $1.5 \%$ isoflurane in oxygen/air (100/400 $\mathrm{mL} / \mathrm{min})$ throughout the measurements. The fur and the scalp over the head of the mice were removed. Animals were placed in prone position on a heating pad that maintains a constant body temperature. Mice were injected intravenously with a $100 \mu$ bolus containing the oxazine probe AOI987 (Fig. 1b, $20.5 \mathrm{mg} / \mathrm{kg}$ body weight, dissolved in $0.1 \mathrm{M}$ phosphate buffer saline (PBS), $\mathrm{pH}$ 7.4) through the tail vein. OA signals were recorded before injection (108 s duration), during injection (432 s duration with i.v. injection starting at $30 \mathrm{~s}$ after 
the beginning of acquisition) and 20, 40, 60, 90 and 120 min post-injection (108 s duration each). The pulse repetition frequency (PRF) of the laser was set to $25 \mathrm{~Hz}$ and the laser wavelength was tuned between 550 and $680 \mathrm{~nm}(5 \mathrm{~nm}$ step) on a per pulse basis. After the in vivo measurements, mice were perfused with $0.1 \mathrm{M}$ PBS ( $\mathrm{pH}$ 7.4) under ketamine/xylazine/acepromazine maleate anesthesia (75/10/2 mg/kg body weight, i.p. bolus injection) and decapitated. The mouse brains were removed from the skull and imaged ex vivo with the vMSOT system. For this, the spherical array was positioned pointing upwards and filled with agar gel to guarantee acoustic coupling, which served as a solid platform to place the excised brains. Uniform illumination of the brain surface was achieved by inserting three arms of the fiber bundle in the lateral apertures of the array and a fourth one providing light delivery from the top. The PRF of the laser was set to 25 $\mathrm{Hz}$ and the laser wavelength was tuned between 420 and $680 \mathrm{~nm}$ (5 nm step) on a per pulse basis. All recorded OA signals were normalized with the calibrated wavelength-dependent energy of the laser pulse. After the ex vivo measurement, mouse brains were fixed in $4 \%$ paraformaldehyde in 0.1 M PBS (pH 7.4) for one day and stored in 0.1 M PBS (pH 7.4) at $4{ }^{\circ} \mathrm{C}$.

\section{vMSOT image reconstruction and multi-spectral analysis}

vMSOT images were displayed in real time during the acquisition procedure. This was achieved by reconstructing the images with a graphics processing unit (GPU)-based implementation of a back-projection formula ${ }^{42}$. More accurate image reconstruction was performed off-line. For this, a 3D model-based (iterative) reconstruction algorithm was used, which has been shown to render more quantitative results ${ }^{43}$. Prior to reconstruction, the collected signals were band-pass filtered with cut-off frequencies 0.1 and $9 \mathrm{MHz}$. The reconstructed images were further processed to unmix the bio-distribution of AOI987 via per-voxel least square fitting of the OA spectral 
profiles to a linear combination of the absorption spectra of oxygenated hemoglobin $\left(\mathrm{HbO}_{2}\right)$, deoxygenated hemoglobin $(\mathrm{Hb})$ and AOI98 $7^{44}$. The OA spectra of the probe was experimentally determined as the average spectra of the differential OA image during bolus perfusion at several major vessels in the brain (Fig. 1b). The OA spectrum of AOI987 approximately matches the absorption spectrum measured with a spectrophotometer (Avantes BV, Apeldoorn, The Netherlands), being the differences arguably due to the fluorescence of the probe ${ }^{45}$. The OA spectra of $\mathrm{Hb}$ and $\mathrm{HbO}_{2}$ was taken from an online database ${ }^{46}$. The displayed single-wavelength and unmixed images were processed with a median filter with a kernel size $3 \times 3 \times 3$.

\section{Co-registration with MRI and volume-of-interest analysis}

The annotated Allen brain atlas ${ }^{47}$ was used to identify brain regions in the vMSOT datasets. For this, the reference atlas was manually aligned with the $600 \mathrm{~nm}$ vMSOT images, which provided the best contrast for the structure of the cortical vessels of the brain. The same transformation matrix was then applied to the unmixed AOI987 images. Subsequently, these images were coregistered with $\mathrm{T}_{2}$-weighted structural MRI images (Ma-Benveniste-Mirrione- $\mathrm{T}_{2}{ }^{48}$ ) from PMOD (now Bruker). Volume-of-interest (VOI) analysis of 15 brain regions was performed using the embedded Mouse VOI atlas (Ma-Benveniste-Mirrione) in PMOD. Specifically, time course of regional AOI987 absorbance (a.u.) and retention (average of 60-120 min) were calculated. Extracranial background signal was removed with a mask from the VOI atlas.

\section{Fluorescence microscopy system}

A previously established hybrid vMSOT and epi-fluorescence system ${ }^{49}$ was employed to compare the time course in one arc $\mathrm{A} \beta$ mouse simultaneously pre- and post $i . v$. bolus injection of 
AOI987. The laser beam was coupled into the system to provide both the illumination for vMSOT and excitation light for fluorescence imaging. The generated acoustic signal was detected by the ultrasonic matrix array detector and then reconstructed as aforementioned. The excited fluorescence signal was collected by an image guide comprised of 100,000 fibers and then imaged onto an EMCCD camera (Andor Luca R, Oxford Instruments, UK). OA and fluorescence signals were recorded before injection, 20, 40, 60, 90 and $120 \mathrm{~min}$ after the injection.

\section{Brain tissue collection, Staining, confocal imaging and selective plane illumination}

\section{microscopy}

Two arcA $\beta$ mice and two non-transgenic littermates were perfused using hydrogel and cleared using a modified electrophoretic clearing chamber for CLARITY ${ }^{50,51}$. The detailed procedure for $\mathrm{A} \beta$ staining using LCOs (hFTAA and qFTAA) and analysis methods for mesoscopic selective plane illumination microscopy (mesoSPIM) ${ }^{52}$ are provided as Suppl materials. Paraffin fixed brains from arcA $\beta$, APP/PS1 and non-transgenic littermate mice were cut horizontally at $5 \mu \mathrm{m}$ thickness. Brain sections were stained with anti-A $\beta$ antibody 6E10, AOI987 and fibrillary conformation anti-amyloid antibody OC (details in Suppl Table 1) as previously described ${ }^{38}$. Nuclei were counterstained by 4',6-diamidino-2-phenylindole (DAPI) while adjacent sections were stained with hematoxylin and eosin $(\mathrm{H} \& \mathrm{E})$. The slices were imaged at 20× magnification using Pannoramic 250 (3D HISTECH, Hungary) and analyzed using ImageJ (NIH, United States). 
The co-localization of the probe AOI987 was evaluated by double staining using antibody $6 \mathrm{E} 10$ and OC to amyloid deposits, and confocal microscopy imaging of the cortex, hippocampus and thalamus of $\operatorname{arcA} \beta, A P P / P S 1$ and non-transgenic littermate mice at $\times 10, \times 63$ magnification using a Leica SP8 confocal microscope (Leica Microsystems GmbH, Germany) at ScopeM ETH Zurich Hönggerberg core facility. Sequential images were obtained by using 405, 488, $650 \mathrm{~nm}$ lines of the laser to excite stained images respectively. Identical settings resolution with $\mathrm{Z}$ stack $(n=15)$ and gain were used. The size of the plaque detected by AOI987 and 6E10 were assessed using full width half maximum (FWHM) method. Double staining using AOI987 and LCOs (hFTAA and qFTAA) were performed to investigate the overlap in detecting $\mathrm{A} \beta$ in the mouse brain slices (Suppl Table 1).

\section{Statistics}

Group comparison of AOI987 absorbance and immunostaining in multiple brain regions was performed by using two-way ANOVA with Turkey's post hoc analysis (Graphpad Prism, Zurich, Switzerland). All data are presented as mean \pm standard deviation. Significance was set at ${ }^{*} p<$ 0.05 .

\section{Results}

\section{In vivo vMSOT amyloid imaging and spectral unmixing}

The novel vMSOT system was specifically arranged to cover the entire mouse brain within the FOV of the transducer array (Fig. 1a), approximately $10 \times 10 \times 10 \mathrm{~mm}^{3}$. The spatial resolution in a region close to the center of the array is $\sim 110 \mu \mathrm{m}$. OA signals are generated by endogenous oxygenated $\left(\mathrm{HbO}_{2}\right)$ and deoxygenated $(\mathrm{Hb})$ hemoglobin as well as by the extrinsically 
administered AOI987 probe. Light absorption in hemoglobin enables distinguishing cerebral vascular structures in the vMSOT images, which serve as an anatomical reference to differentiate different brain regions. The absorption spectra of $\mathrm{HbO}_{2}$ and $\mathrm{Hb}$ are displayed in Fig. 1b. It is shown that hemoglobin absorption strongly decays for wavelengths larger than $600 \mathrm{~nm}$. This decay negatively affects the signal-to-noise ratio of the vMSOT images, but on the other hand enables deeper light penetration. The OA image at $600 \mathrm{~nm}$ was found to represent a good tradeoff between signal strength and light attenuation for an optimal contrast of the cortical brain vasculature. The maximum intensity projection of the OA image at $600 \mathrm{~nm}$ along the depth direction is displayed in Fig. 1c, where major vessels and brain areas are labelled (Suppl video 1). The OA absorbance spectrum of AOI987 derived from in vivo vMSOT imaging data in the brain from arcA $\beta$ and APP/PS1 mouse brains is also shown in Fig. 1b along with the absorbance measured with a spectrometer (see methods section). The OA spectrum is characterized by a major peak at $650 \mathrm{~nm}$ and minor peak at $600 \mathrm{~nm}$. Considering that strong difference in optical attenuation are produced by light absorption in hemoglobin, only wavelengths above $600 \mathrm{~nm}$ were considered for spectral un-mixing. A 3D view of the un-mixed image for AOI987 corresponding to $60 \mathrm{~min}$ after injection of the probe is shown SFig. 1 and Suppl video 2 superimposed to the anatomical image of the brain acquired with MRI (Fig. 1d). The same 3D view additionally including the OA image at $600 \mathrm{~nm}$ as an anatomical reference is also displayed in Fig. $1 d$.

The accuracy of multispectral un-mixing was assessed by considering the sequence of images acquired during injection of the probe in an $\operatorname{arc} \mathrm{A} \beta$ transgenic mouse. In this case, the biodistribution of the probe could alternatively be un-mixed by considering the difference with 
respect to the reference image before injection. For this, the average of 25 OA images corresponding to $650 \mathrm{~nm}$ excitation was taken. A comparison of the bio-distribution of AOI987 estimated via difference with respect to the background and via multi-spectral un-mixing is shown in Figs. 1d, g. Specifically, one cross-sections of the vMSOT images are displayed superimposed to the corresponding MRI sections (Figs. 1f, i). After i.v. bolus injection of AOI987 through the mouse tail vein, an OA signal increase was observed in the mouse brain. Immediate uptake was observed in major vessels (superior sagittal sinus and large vessel at the cortical surface), for which the intensity is higher than that in the brain tissue (e.g. in the cortex and hippocampus). The good match between the two un-mixing approaches was further corroborated by considering the time dynamics of the un-mixed signals at specific locations labelled in Fig. 1d, g. All the selected profiles showed similar patterns during the $432 \mathrm{~s}$ image acquisition window (Figs. 1e, h). This validates the results obtained with multi-spectral unmixing, which is the only available method for other measurements. 

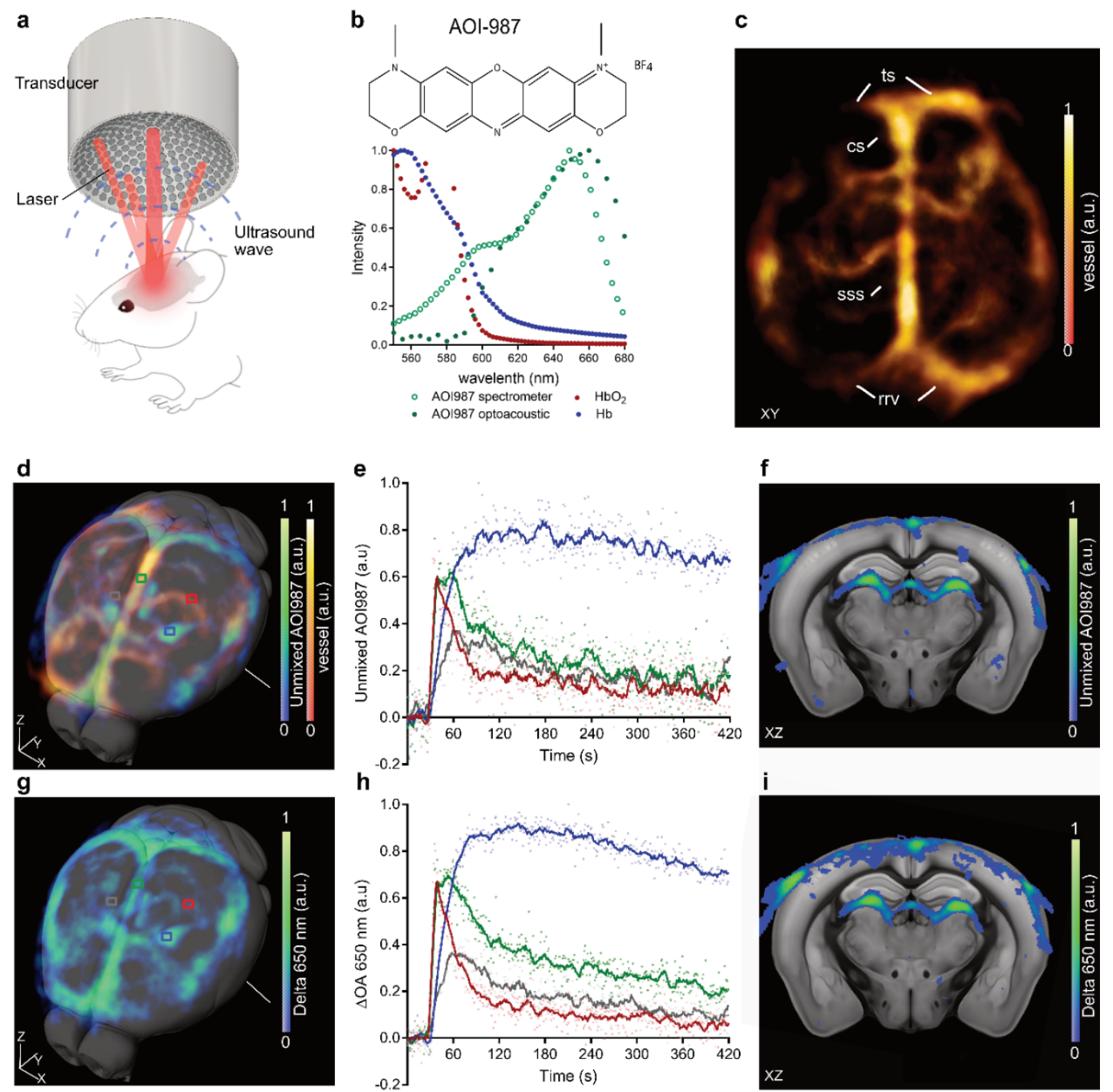

Figure 1. In vivo volumetric multispectral optoacoustic tomography (vMSOT) method. a) set-up of vMSOT; b) absorbance spectrum of AOI987 measured by using vMSOT and using spectrometer; c) horizontal view of in vivo vMSOT data, vasculature $\left(\mathrm{Hb} / \mathrm{HBO}_{2}\right.$, red-yellow); confluence of sinuses (cs), rostral rhinal vein (rrv), superior sagittal sinus (sss), transverse sinus (ts); d) 3D rendering of vMSOT data and overlay on MRI structural data with vMSOT signals unmixed for $\mathrm{Hb} / \mathrm{HbO}_{2}$ corresponding to the vasculature, unmixed for AOI987. AOI987 are indicated in blue-green and $\mathrm{Hb} / \mathrm{HbO}_{2}$ in red-yellow; g) 3D rendering of vMSOT data and overlay on MRI structural data with vMSOT signals for delta absorbance at $650 \mathrm{~nm}$ in blue-green; e) unmixed AOI987 absorbance; and $\mathbf{h}$ ) delta absorbance at $650 \mathrm{~nm}$ from dynamic vMSOT over 07 minutes in $\operatorname{arc} \mathrm{A} \beta$ mouse brain. AOI987 injected i.v. at 30 seconds. The changes in unmixed AOI987 absorbance and delta absorbance at $650 \mathrm{~nm}$ was analyzed in four different brain regions (cortex (red), superior sagittal sinus (blue), hippocampus (green), vessel (grey) on the cortical surface indicated in $\mathbf{d}, \mathbf{g}$ ); f) unmixed AOI987 absorbance and $\mathbf{h}$ ) delta absorbance at $650 \mathrm{~nm}$ in $\operatorname{arc} \mathrm{A} \beta$ mouse brain in one coronal slices and overlay on MRI structural data (anatomical levels are indicated in $\mathbf{d}$ and $\mathbf{g}$ ). 


\section{AOI987 optoacoustic signal retention in the brain of $\operatorname{arcA} \beta$ and APP/PS1 models}

The retention of AOI987 was first assessed in the vMSOT and epi-fluorescence images of an $\operatorname{arcA} \beta$ mouse acquired simultaneously using hybrid system ${ }^{49}$. Similar temporal profiles the vMSOT and fluorescence signals of the cortical region were observed (Fig. 1). Higher retention at 120 min post-injection in the cortex compared to the cerebellum was observed with both imaging systems, suggesting the specific binding of AOI987 in the cortical deposits. The ratio of fluorescence intensity in the cortex/cerebellum at $120 \mathrm{~min}$ post-injection was approximately 1.4 in fluorescence microscopy images taken ex vivo.

A more comprehensive evaluation of the bio-distribution of the probe was then performed. Three $\operatorname{arcA} \beta$ mice, three APP/PS1 mice and six non-transgenic littermates were scanned in vivo by using vMSOT at several time points until 120 min following AOI987 injection (Fig. 2a-c). Multispectral unmixed image of AOI987 channel, and atlas based volume-of-interest analysis were performed (Fig. 2d). Two-way ANOVA with Tukey's post hoc analysis showed the interaction between brain region and genotype. Significantly higher AOI987 retention (average of absorbance at 60-120 min post-injection) were observed in the cortex $(\mathrm{p}=0.0002)$, hippocampus $(\mathrm{p}=0.0134)$ and thalamus $(\mathrm{p}<0.0001)$ of $\operatorname{arc} \mathrm{A} \beta$ mice compared to non-transgenic littermates. Only in the cortex $(\mathrm{p}=0.0016)$ of APP/PS1 mice higher AOI987 retention was observed compared to non-transgenic littermates. No apparent accumulation in the brainstem was observed in neither of the transgenic mice. ANNOVA for repeated measure Fig. $\mathbf{2 f}$-j showed the time course of AOI987 absorbance in different brain regions of arcA $\beta$, APP/PS1 mice and non-transgenic littermates. The signals were highest in the cortex and thalamus followed by 
subcortical brain regions and cerebellum and were absent in the brain stem. Fluorescence intensity: F.I.
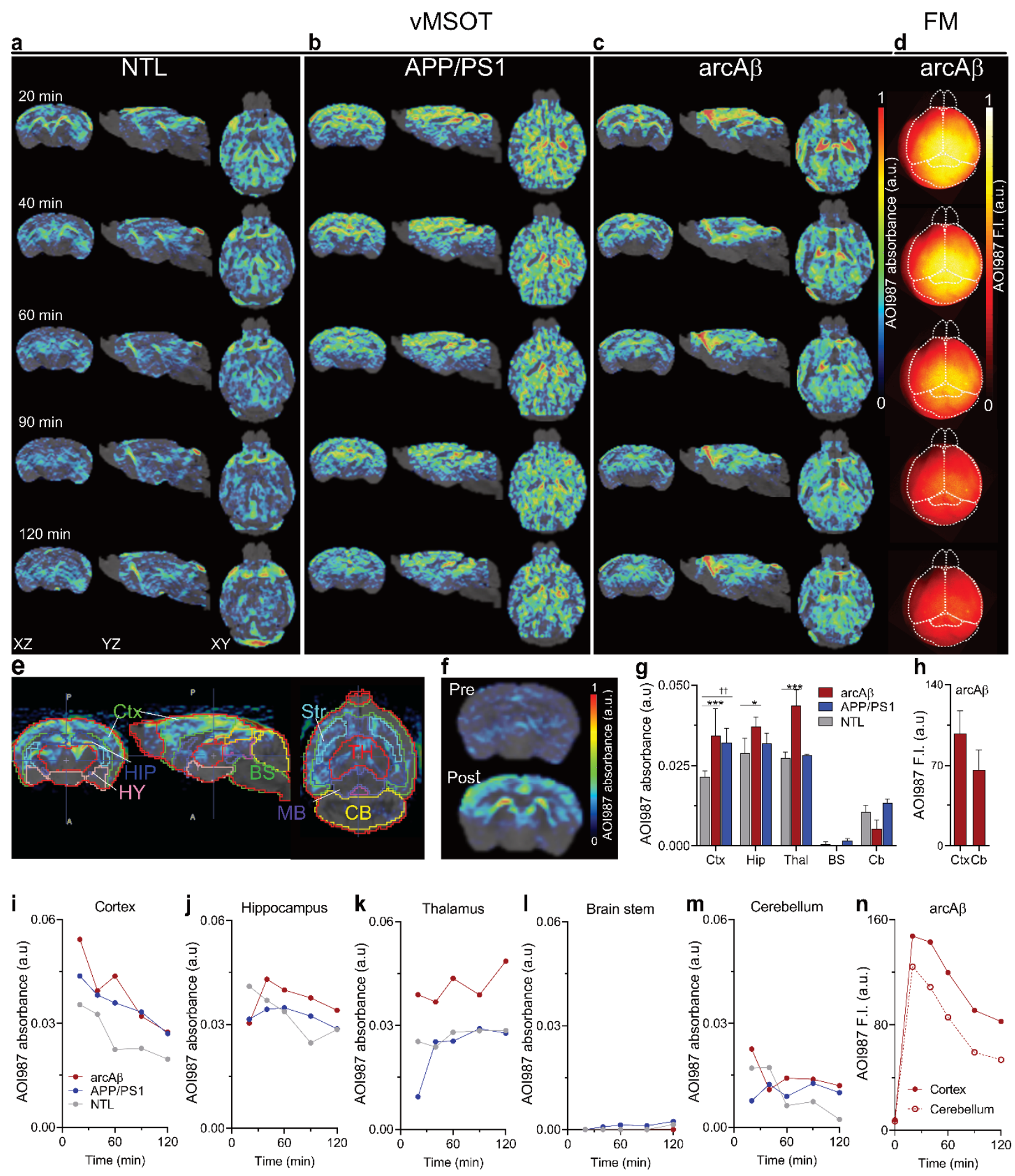

Figure 2. Regional brain AOI987 distribution revealed by using in vivo volumetric multispectral optoacoustic tomography (vMSOT) with AOI987 in arcA $\beta$ and APP/PS1 
mice. vMSOT in a) non-transgenic littermates (NTLs), b) transgenic APP/PS1 mice and c) $\operatorname{arcA} \beta$ mice; at 20, 40, 60, 90 and 120 min following dye administration showing coronal, sagittal and horizontal views overlaid over the corresponding masked magnetic resonance imaging -based brain atlas. AOI987 absorbance (a.u.) are indicated in rainbow color; d) fluorescence microscopy (FM) in one arcA $\beta$ mouse at 20, 40, 60, 90 and 120 min following dye administration showing horizontal view; e) volume-of-interest used for quantitative analysis of regional AOI987 retention; f) pre- and 20 min post dye injection in one arcA $\beta$ mouse brain, unmixed, coronal view; g) regional comparison of averaged AOI987 absorbance (a.u.) retention at 60-120 min post-injection; h) quantification of AOI987 fluorescence intensity in the cortex and cerebellum; i-m) time course of cortical, hippocampal, thalamic, brain stem and cerebellar volume-of-interest AOI987 signal (a.u.) in NTL, $\operatorname{arcA} \beta$ and APP/PS1 mice; n) time course of cortical and cerebellar region-of-interest fluorescence intensity (a.u.) in one arcA $\beta$ mouse. Brain stem: BS; Cortex: Ctx; Cerebellum: Cb; Hippocampus: Hip; Hy: Hypothalamus; Midbrain MB; Thalamus: TH; $* p<0.05$; *** $p<0.001$ comparison between NTL and arcA $\beta$ mice, and ${ }^{\dagger \dagger} p<$ 0.01 comparison between NTL and APP/PS1 mice; two-way ANOVA with post-hoc Tukey's analysis. All data are present as mean \pm standard deviation.

\section{Ex vivo vMSOT imaging of the mouse brain}

Ex vivo vMSOT imaging was performed on PBS perfused (blood washed out) mouse brains excised after in vivo imaging. The acquired images served to validate the in vivo AOI987 signal bio-distribution in a scenario not affected by cross-talk un-mixing artefacts associated to the presence of $\mathrm{Hb}$ and $\mathrm{HbO}_{2}$ in a living animal. The same image reconstruction and multi-spectral un-mixing methods used for the analysis of the in vivo datasets were considered. The background tissue provide sufficient contrast in the vMSOT for structural information, and hence was used for regional analysis of AOI987 signal in the mouse brain. Figs. 3a-b display a comparison of the 3D unmixed AOI987 signal overlaid with structural signal $(600 \mathrm{~nm})$ from one non-transgenic mouse and one $\operatorname{arcA} \beta$ mouse brain. The accumulation of AOI987 signal in the cortex, hippocampus and thalamus of $\operatorname{arc} \mathrm{A} \beta$ mouse suggest specific binding of the probe as the regions are known to express high plaque load ${ }^{37}$. In contrast, little signals attributed to AOI987 have been observed in the brain of a non-transgenic littermate (Fig. 3c, SFig. 2). 

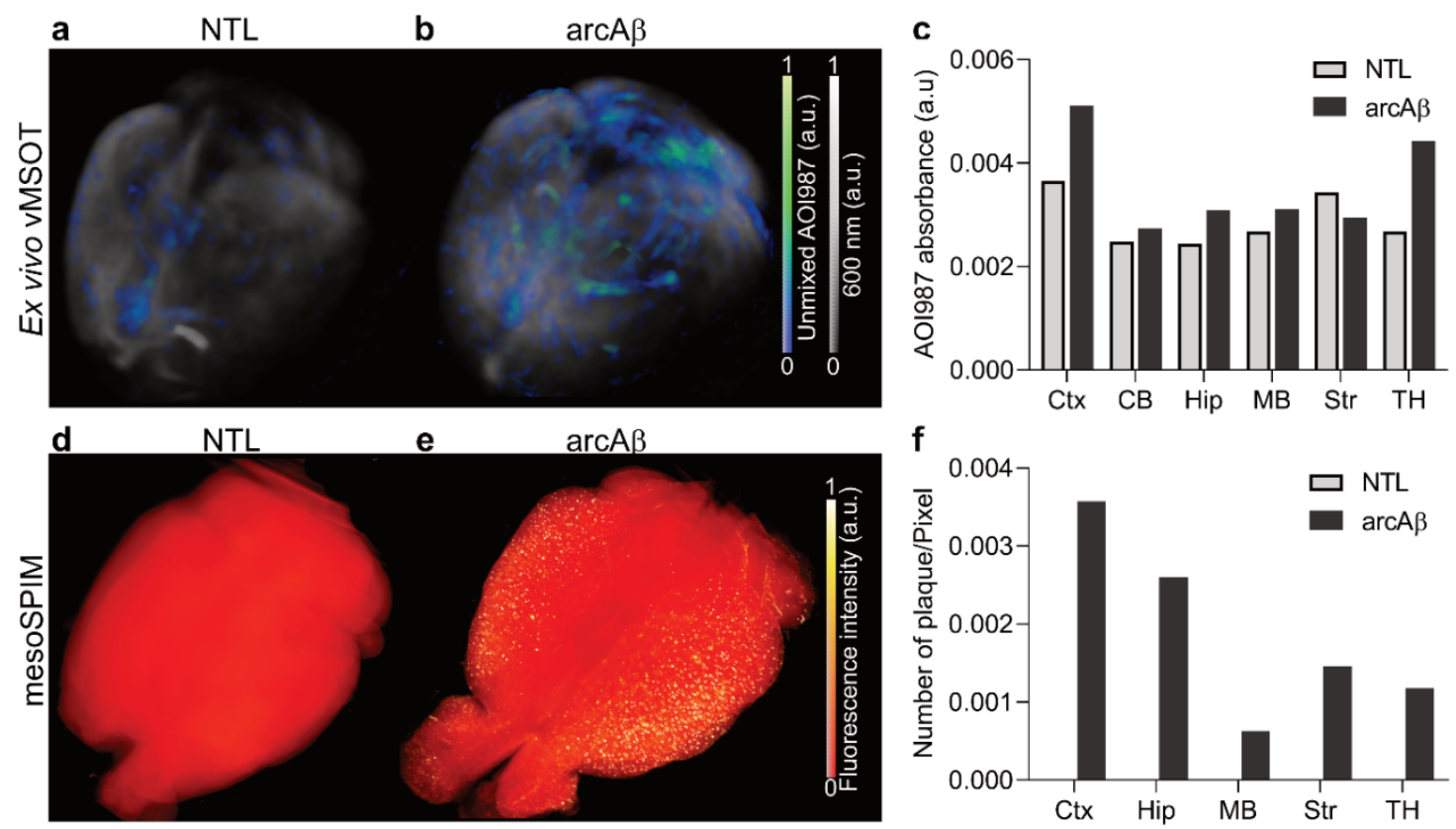

Figure 3. Ex vivo volumetric multispectral optoacoustic tomography (vMSOT) imaging and whole brain light-sheet microscopy using mesoSPIM of brain from transgenic $\operatorname{arcA} \beta$ and non-transgenic littermate (NTLs) mice. a) 3D rendering of vMSOT data overlay of vMSOT structural information with vMSOT signals unmixed for AOI987 in one non-negative control mouse; and b) one arcA $\beta$ mouse; AOI987 are indicated in blue-green; $600 \mathrm{~nm}$ vMSOT signal provide structural information (a.u.) of the brain at ex vivo; c) Quantification of ex vivo regional AOI987 absorbance (a.u.) in NTL and $\operatorname{arcA} \beta$ mouse brain; d) 3D rendering of mesoSPIM data of amyloid-beta distribution (stained by using luminescent conjugated oligothiophene) in one NTL mouse; and e) one arc $\mathrm{A} \beta$ mouse. f) Quantification of regional fluorescence intensity in NTL and $\operatorname{arcA} \beta$ mouse brain. Cortex: Ctx; Hippocampus: Hip; Midbrain: MB; Striatum: Str; Thalamus: TH.

\section{MesoSPIM for mapping amyloid distribution in the whole brain of $\operatorname{arcA} \beta$ mouse}

MesoSPIM ${ }^{52}$ has been used to determine the 3D distribution of AOI987 in brains of arcA $\beta$ mice and non-transgenic littermates with luminescent conjugated oligothiophene probe. SPIM imaging of the cleared brain of arc $\mathrm{A} \beta$ mice revealed signals in the cortical, hippocampal and thalamic parenchyma as well as in vascular structures, indicative of aggregated $A \beta$ accumulation. In contrast, brains of non-transgenic littermates displayed only background signal intensity with the exception of few high intensity signal attributed to vessels (Figs. 3d-e, SFig. 3 and Suppl video. 
3). Regionally resolved quantitative analysis of numbers of amyloid deposits revealed a higher load in the cortical, hippocampus and thalamic regions-of-interest as compared to cerebellar and other subcortical regions (Fig. 3f, Suppl materials). Regions were defined on the basis of the Allen brain atlas. Double staining using AOI987 and hFTAA shows predominantly overlap in the detection of both vascular and parenchymal plaque in the $\operatorname{arcA} \beta$ mouse brain (SFigs. 4e-k), whereas less convergence between AOI987 and qFTAA (SFigs. 4a-d).

\section{Immunohistochemical staining and confocal microscopy}

Immunohistochemical staining were performed on horizontal brain tissue sections from $\operatorname{arcA} \beta$, APP/PS1 mice and non-transgenic littermates using AOI987, 6E10, OC and counterstained using DAPI (Fig. 4). In APP/PS1 mice, the A $\beta$ plaques were predominantly observed in the parenchyma, most pronounced in the cortex (Figs. 4i-j) and hippocampus (Figs. 4e-f). In $\operatorname{arcA} \beta$ mice, the 6E10 and AOI987 stained A $\beta$ deposits were distributed both in the cortical (Figs. 4k-l), hippocampal (Figs. 4g-h) and thalamic (Figs. 4o-p) parenchyma as well as along vascular structures predominantly in the cortex. The staining distribution reveals that AOI987 and 6E10 interact with both parenchymal and vascular amyloid deposits, the latter being indicative of cerebral amyloid angiopathy. The plaques appeared less diffuse in $\operatorname{arc} \mathrm{A} \beta$ mice compared to that in APP/PS1 mice. The size of deposits in the cerebral cortex stained by AOI987 tends to be smaller than that stained by $6 \mathrm{E} 10$, the difference being more pronounced in APP/PS1 than in the $\operatorname{arcA} \beta$ mice (Fig. 4u). For smaller plaques, AOI987 and 6E10 almost fully overlapped (Fig. 4q). Similarly, there was a good correspondence of OC stained fibrillar A $\beta$ with AOI987 in the cortical regions-of-interest of APP/PS1 mice (Fig. 4r) and $\operatorname{arcA} \beta$ mice (Figs. 4s-t). No signal of 
bioRxiv preprint doi: https://doi.org/10.1101/2020.02.25.964064; this version posted February 25, 2020. The copyright holder for this preprint (which was not certified by peer review) is the author/funder, who has granted bioRxiv a license to display the preprint in perpetuity. It is made available under aCC-BY-NC-ND 4.0 International license.

AOI987 was detected in the brain sections from non-transgenic littermates as there is no amyloid plaque (Figs. 4v-w). 
bioRxiv preprint doi: https://doi.org/10.1101/2020.02.25.964064; this version posted February 25, 2020. The copyright holder for this preprint

(which was not certified by peer review) is the author/funder, who has granted bioRxiv a license to display the preprint in perpetuity. It is made available under aCC-BY-NC-ND 4.0 International license.

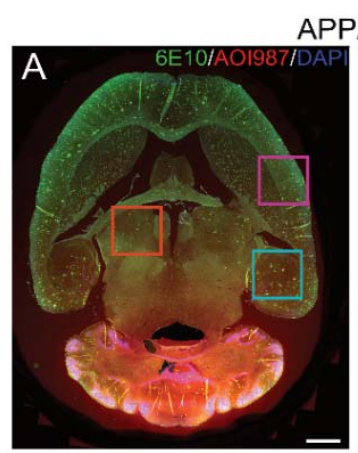

APP/PS1
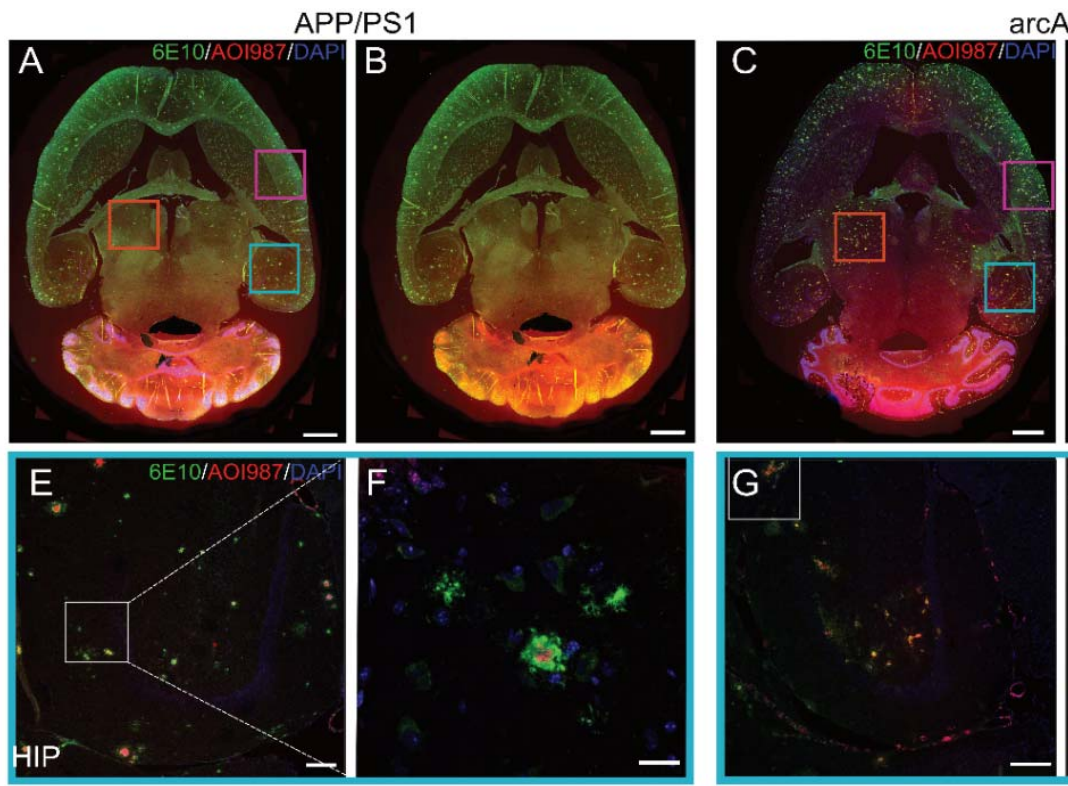

\section{$\operatorname{rcA} \beta$}
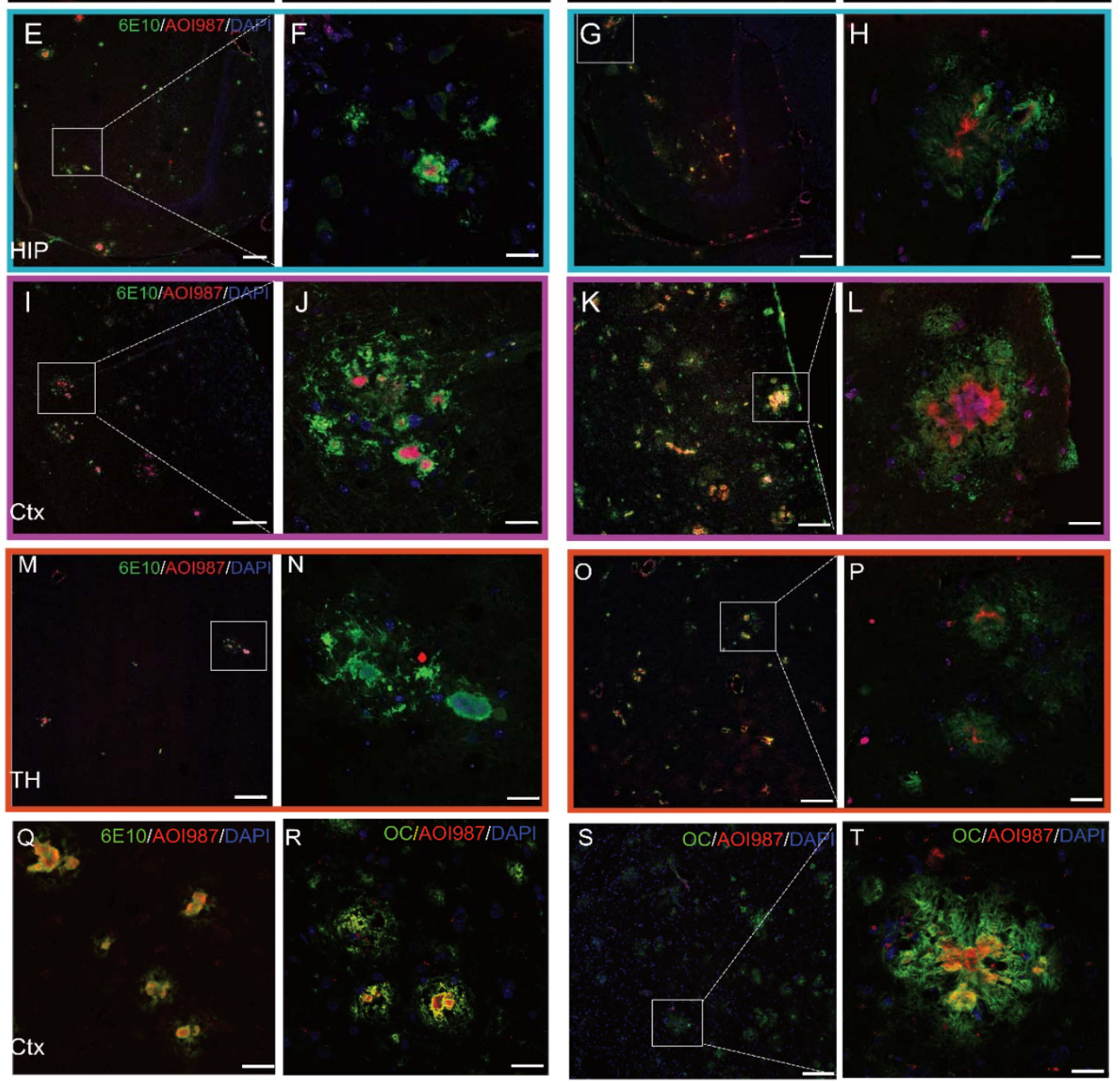

U
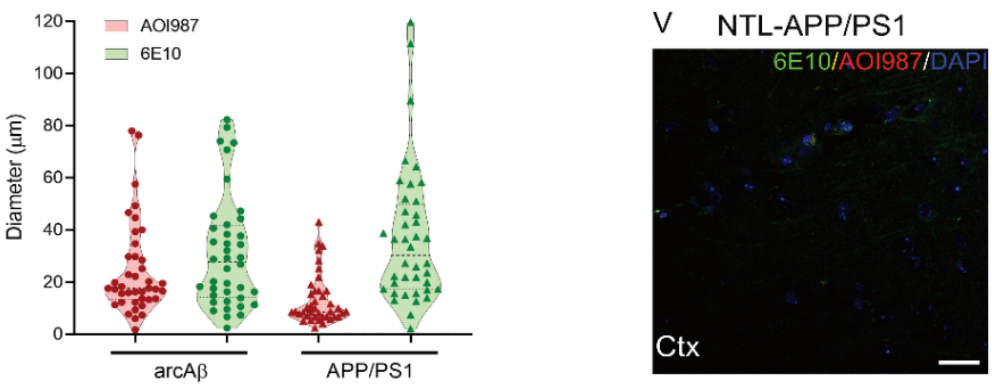

W NTL-arcA $\beta$

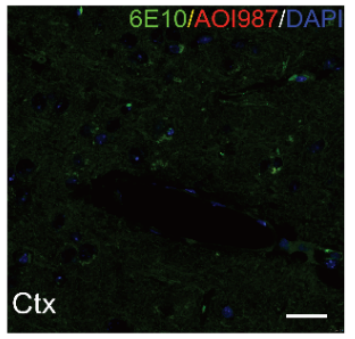


Figure 4. Staining for $A \beta$ deposition in APP/PS1 and $\operatorname{arcA} \beta$ mouse brain tissue sections. (a, b) confocal microscopic images of horizontal whole brain sections from APP/PS1 mouse; and (c, d) from $\operatorname{arcA} \beta$ mouse; DAPI (blue), Alexa488-6E10 (green), AOI987 (red); Zoomed-in images are shown for hippocampus (e-h, blue square in $(\mathbf{a}, \mathbf{c})$ ); cortex (i-l, $\mathbf{q}, \mathbf{s}$ magenta square in (a, c)) and thalamus (m-p, orange square in $(\mathbf{a}, \mathbf{c})$ ), respectively, demonstrating the co-localization of 6E10 and AOI987 to amyloid-beta plaque in APP/PS1 and $\operatorname{arcA} \beta$ mouse brain with further magnification of selected regions-of-interest (white squares). DAPI (blue), Alexa488-6E10 (green), AOI987 (red); r-t) Confocal microscopic images of cortical section from APP/PS1 and $\operatorname{arcA} \beta$ mice indicates co-localization of OC targeting amyloid fibrils and AOI987 stained deposits, OC (green), AOI987 (red), DAPI (blue); Again, volume of AOI987 stained structures appears smaller than OC stained ones; (u) size analysis indicates that stained volume of cortical parenchymal plaques was larger when using $6 \mathrm{E} 10$ (targeting $\mathrm{A} \beta_{1-16}$ ) as compared to AOI987 stains for both APP/PS1 and $\operatorname{arcA} \beta$ mice; $(\mathbf{v}, \mathbf{w})$ confocal imaging in horizontal cut cortex from non-transgenic littermate mice, DAPI (blue), Alexa488-6E10 (green), AOI987 (red). Scale bar $=1 \mathrm{~mm}(\mathbf{a}-\mathbf{d}) ; 100 \mu \mathrm{m}(\mathbf{e}, \mathbf{g}, \mathbf{i}, \mathbf{k}, \mathbf{m}, \mathbf{o}, \mathbf{s}) ; 20 \mu \mathrm{m}(\mathbf{f}, \mathbf{h}, \mathbf{j}, \mathbf{l}, \mathbf{n}, \mathbf{p}, \mathbf{r}, \mathbf{t}, \mathbf{v}, \mathbf{w})$ respectively.

\section{Discussion}

The development of new tools for non-invasive detection of $A \beta$ deposits with high resolution in animal models of $\mathrm{AD}$ is critical for a better understanding of the disease mechanisms and translational development of $\mathrm{A} \beta$-targeted therapies. We developed a vMSOT system used previously for $\mathrm{FONT}^{32,35}$, for fast 3D whole brain amyloid imaging. AO1987, an A $\beta$-targeting probe 26, 27, for optoacoustic imaging in two different models of AD amyloidosis. Fast volumetric acquisition of multispectral optoacoustic tomographic data together with a novel spectral unmixing approach and brain atlas-based data co-registration allowed us to systematically quantify brain-wide distribution of $A \beta$ plaque pathology at mesoscale resolution. The comparison of two commonly used models of $\mathrm{AD}$ amyloidosis with vMSOT enabled to reveal strain-specific pattern of $A \beta$ deposition.

So far, at high- resolution imaging of $\mathrm{A} \beta$ load across the whole brain is only possible ex vivo by histopathological and microscopic techniques ${ }^{13,14}$ that require extensive tissue processing and 
are both painstaking and time-consuming. The vMSOT employed in this work is capable of imaging the entire mouse brain at mesoscale resolution $(\sim 110 \mu \mathrm{m})$ and is sensitive to optical contrast agents targeting $\mathrm{A} \beta$. Herein, we demonstrated that 3D vMSOT imaging assisted with the amyloid-binding probe AOI987 accurately maps A $\beta$ deposits across the murine brain in two transgenic mouse models of cerebral amyloidosis commonly used in $\mathrm{AD}$ research. In $\operatorname{arc} \mathrm{A} \beta$ mice intracellular punctate $A \beta$ deposits start concomitantly with robust cognitive impairments at the age of 6 months before the onset of $A \beta$ plaque formation and cerebral amyloid angiopathy ${ }^{36}$. In APP/PS1 mice, the A $\beta$ deposits accumulate earlier and mainly in parenchyma ${ }^{41}$. We found that vMSOT mapping of AOI987 specifically detected high cortical, hippocampal and thalamic retention in $\operatorname{arc} \mathrm{A} \beta$ mice, while only higher cortical retention in APP/PS1 mice. The in vivo $\mathrm{A} \beta$ distribution patterns revealed with vMSOT were shown to be in accordance with our immunohistochemical staining results and the known $\mathrm{A} \beta$ distribution in both transgenic mouse models 36,41 .

The limitations of ex vivo $\mathrm{A} \beta$ assessment techniques has spurred the search for $\mathrm{A} \beta$ imaging techniques. PET would be the method of choice, as it would directly facilitate translational studies assessing $A \beta$ in $A D$ patient studies ${ }^{6,7,8,109,10}$ and animal models ${ }^{21} 2223$. In transgenic models PET amyloid imaging is limited as currently achievable microPET for small animal achieves $\sim 1 \mathrm{~mm}$ resolution. vMSOT provides $\sim 100 \mu \mathrm{m}$ spatial resolution which is $10 \times$ higher. This becomes significant when $A \beta$ load is assessed in different brain regions. While PET can only coarsely discriminate between cortical and subcortical structures, novel vMSOT can assess A $\beta$ accumulation in different regions. This advantage helps to identify mouse strain-specific pattern of $A \beta$ deposition and to study the spread of $A \beta$ across the brain over the disease course. 
vMSOT further covers a larger range of spatial scales than pure optical methods, which either lack the penetration depth (i.e. optical microscopy) or the spatial resolution (i.e. diffuse optical imaging and tomography) ${ }^{29}$. AO1987 has been used in NIRF imaging and FMT ${ }^{26,27}$ for detecting amyloid pathologies in an APP23 mouse model of AD cerebral amyloidosis. In NIRF imaging, the fluorescence signal that is quantified over the brain regions contains also other signal contribution from superficial tissues, i.e. skin, muscle and skull, rending quantification difficult. Indeed, biological tissues are highly scattering and light propagation is mainly diffusive for depths beyond $1 \mathrm{~mm}$. Although mathematical models based on diffuse optics are available e.g. for $\mathrm{FMT}^{27}$, quantification is generally not possible in the diffuse regime of light. In contrast, due to the true 3D mapping capability of vMSOT, the photoacoustic signal can be quantified for the different compartments provided proper image reconstruction and processing is performed. Thus, the results obtained indicate that the unprecedented capabilities provided empower vMSOT as a new method that can clearly outperform existing in vivo amyloid imaging technologies in animal models.

In the current implementation, a linear un-mixing algorithm was used for calculating the biodistribution of AOI987 in deep brain regions such as the hippocampus and the thalamus. However, it is noted that the wavelength-dependent optical attenuation causes distortion in the OA spectra of signals generated at deep locations (spectral colouring), while the non-uniform laser beam shape further affects the superficial signals. These and other effects lead to cross-talk artefacts in the un-mixed image corresponding to the contrast agent of interest, so that more advanced algorithms are required for an optimal performance. The good correspondence between 
the in vivo and ex vivo regional bio-distribution however suggests that this un-mixing approach is generally suitable for our purposes. The reconstruction algorithm is also essential for quantifying the concentration of the probe. The model-based algorithm employed to reconstruct the images off-line has been shown to provide more quantitative results than standard back-projection algorithms. However, acoustic distortions associated to speed of sound heterogeneities, acoustic scattering and attenuation as well as the response of the ultrasound sensing elements of the array are known to additionally play a role in the quality of the images rendered. More accurate estimation of the bio-distribution of the contrast agent of interest can also be facilitated with pharmacokinetic modeling, which has been previously developed based on the anatomical information in cross-sectional MSOT images ${ }^{53,54}$. Registration between MSOT and MRI/atlas can additionally boost the performance of these models and further provide a better anatomical reference for regional analysis. Automatic registration methods have been established for 2D dataset $^{55,56}$, which can potentially be adapted to $3 \mathrm{D}$ vMSOT datasets. However, as both $\operatorname{arcA} \beta$ and APP/PS1 mice showed amyloid deposits in the cerebellum at an old age, regions typically considered as reference region, we therefore opted for average absorbance retention (60-120 min) rather than the reference tissue region modelling. Alternatively, brainstem regions might be considered as reference as they hardly display probe accumulation.

Our approach aimed to provide a consist plaque labeling method using a single A $\beta$-targeted probe and standardized imaging conditions to quantitatively compare plaque density across individual animals and strains. However, we noted that the size of deposits in the cerebral cortex stained by AOI987 was slightly smaller than that stained by $6 \mathrm{E} 10$, the difference being more pronounced in APP/PS1 than in the $\operatorname{arc} \mathrm{A} \beta$ mice. We are still confident that vMSOT does not 
grossly underestimate plaque load, which is similar to what has been reported with histopathology and microscopy ${ }^{16,57}$.

$\mathrm{A} \beta$ deposition in the brain is complex process and involves various molecular species including $\mathrm{A} \beta$ oligomers, $\mathrm{A} \beta$ fibrils, as well as large insoluble aggregates (plaques). A $\beta$ peptides are generated via sequential proteolytic cleavage of the amyloid precursor protein (APP) ${ }^{5}$. Initial cleavage of APP by $\beta$-secretase generates a membrane-bound C-terminal fragment of APP ( $\beta$ $\mathrm{CTF}$ ), which is sequentially processed by $\gamma$-secretase resulting in the formation of longer species, like $A \beta 40$ and $A \beta 42 / 43$, in addition to several other $C$-terminally truncated $A \beta$ variants ${ }^{58}$. A full set of $\mathrm{A} \beta$ isoforms with both $\mathrm{N}$ - and $\mathrm{C}$-terminally truncated $\mathrm{A} \beta$ species can be detected in the post-mortem $\mathrm{AD}$ brain and cerebrospinal fluid ${ }^{59}$. N-terminally truncated $\mathrm{A} \beta$ isoforms show an increased aggregation propensity and have previously been associated with the deposition of dense-core amyloid plaques in the brain ${ }^{60}$. In contrast, C-terminally truncated shorter $A \beta$ isoforms show a higher solubility in vitro and are more commonly detected in the cerebral vasculature ${ }^{61}$. The high degree of heterogeneity in the molecular architecture of misfolded $A \beta$ observed in patients with $\mathrm{AD}$ may be related to the diversity of disease-associated mutations ${ }^{62}$. LCO has been applied to detect $\mathrm{A} \beta \beta$-sheet structures in different animal models of $\mathrm{AD}$ amyloidosis ${ }^{63,64}$ and recognizing structural variants of $\mathrm{A} \beta$ fibrils from brain tissue from $\mathrm{AD}$ patients carrying different mutations ${ }^{62,65}$. Yet the approach is not suited for in vivo applications. Instead, AOI987 has been used due to its strong absorption in the far-red/NIR range warranting efficient tissue penetration of the excitation light, its water solubility and its high binding affinity to $\mathrm{A} \beta$ fibrils ${ }^{26}$. Moreover, the dye has been shown to efficiently cross the blood-brain barrier passing property enabling NIRF imaging and FMTapplications ${ }^{26}$. We observed regional plaque 
density with vMSOT in our study for arcA $\beta$ and APP/PS1 mice. An earlier study compared five mouse lines displaying cerebral amyloidosis, i.e. arcA $\beta$, APP23, APPswe, PSAPP and $\triangle \mathrm{E} 22$ mice for two different PET tracers ${ }^{11} \mathrm{C}$-PIB and ${ }^{18} \mathrm{~F}$-flutemetamol ${ }^{66}$. The differences across mouse lines may arise due to regional differences in $\mathrm{A} \beta$ deposition, due to structural variations of the $A \beta$ species with binding sites exhibiting different affinity to the respective imaging probe, as well as different binding sites for different imaging probes or even several binding sites per $\mathrm{A} \beta$ molecular entity $62,63,65,67,68$. The current in vivo data do not allow discriminating these cases. Further studies will be needed to elucidate if AOI987, PIB and flutemetamol behave differently, i.e. reveal slightly different results regarding the spatial distribution, and different $A \beta$ deposits.

3D amyloid imaging with light sheet microscopy in cleared brain tissues (3DISCO or CLARITY $^{51}$ ) of AD mouse models using anti-A $\beta$ antibodies, Congo-red and methoxy-X04 ${ }^{69}$ has been reported previously. While the method enables imaging at single-cell resolution in the entire brain ${ }^{50}$ it requires extensive tissue processing and lengthy image post-processing limiting the capability of high through-put imaging. Herein, we have used ex vivo light-sheet microscopy to assess the distribution of $\mathrm{A} \beta$ plaques in the $\operatorname{arc} \mathrm{A} \beta$ mouse brain, which confirmed our findings from vMSOT mapping of parenchymal and vascular $A \beta$ deposits. Hence, vMSOT in combination with an $\mathrm{A} \beta$ specific absorbing dye such as AOI987, enables fast volumetric mapping of $A \beta$ distribution across the whole mouse brain in vivo combining the high sensitivity of optical imaging with the high spatial resolution of ultrasound imaging.

In conclusion, we demonstrated the feasibility of quantitative whole brain imaging of the $A \beta$ distribution in mouse models of $\mathrm{AD}$ amyloidosis at high spatial and temporal resolution in vivo 
by vMSOT. Brain MRI atlas was used as a structural reference. In vivo results have been validated using light-sheet microscopy of cleared brain specimen and immunohistology. The vMSOT imaging platform lends itself for longitudinal monitoring of the disease process, its modulation by therapeutic interventions targeting at $\mathrm{A} \beta$, and potentially for studying other proteinopathy (such as tauopathy) in animal models ${ }^{70}$.

\section{Availability of data and material}

The datasets generated and/or analyzed during the current study are available in the repository zenodo DOI 10.5281/zenodo.3576174. The light-sheet microscopy dataset and github code for registration and quantification with Allen brain atlas are available at github (details in Suppl materials).

\section{Declaration of conflict of interests}

No competing interests declared.

\section{Author Contributions}

RN, LD, DR, JK conceived and designed the study; RN, LD, DK performed the experiments; MR provided AOI987; KPRN provided FTAAs. RN, LD, AC, DK analyzed the data; RN, LD, DR and JK interpreted the results; RN, LD, MR, DR, and JK wrote the paper; all coauthors contributed constructively to the manuscript.

\section{Acknowledgement}


The authors acknowledge technical support from Dr. Linjing Mu at Institute of Pharmaceutical

Sciences, ETH Zurich, Dr. Joachim Hohl, Dr. Gabriella Bodizs of the Scientific Center for

Optical and Electron Microscopy (ScopeM) of ETH Zurich; Ms Gloria Shi, Ms Marie Rouault at

Institute for Biomedical Engineering of ETH Zurich; Dr. Luka Kulic, Ms Priyanka Ravikumar at Institute for Regenerative Medicine of University of Zurich for technical help.

\section{Funding}

JK received funding from the Swiss National Science Foundation (320030_179277), in the framework of ERA-NET NEURON (32NE30_173678/1), the Synapsis foundation and the Vontobel foundation. RN received funding from the University of Zurich Forschungskredit (Nr. FK-17-052), and Synapsis foundation career development award (2017 CDA-03).

\section{References}

1. Walsh DM, Selkoe DJ. A critical appraisal of the pathogenic protein spread hypothesis of neurodegeneration. Nature reviews Neuroscience 17, 251-260 (2016).

2. Thal DR, Rub U, Orantes $M$, Braak H. Phases of $A$ beta-deposition in the human brain and its relevance for the development of AD. Neurology 58, 1791-1800 (2002).

3. Hardy J, Selkoe DJ. The amyloid hypothesis of Alzheimer's disease: progress and problems on the road to therapeutics. Science 297, 353-356 (2002).

4. Riek R, Eisenberg DS. The activities of amyloids from a structural perspective. Nature 539, 227235 (2016).

5. Selkoe DJ, Hardy J. The amyloid hypothesis of Alzheimer's disease at 25 years. EMBO Mol Med, (2016).

6. Klunk WE, et al. Binding of the positron emission tomography tracer Pittsburgh compound-B reflects the amount of amyloid-beta in Alzheimer's disease brain but not in transgenic mouse 
brain. The Journal of neuroscience : the official journal of the Society for Neuroscience 25, 1059810606 (2005).

7. Curtis $C$, et al. Phase 3 trial of flutemetamol labeled with radioactive fluorine 18 imaging and neuritic plaque density. JAMA Neurol 72, 287-294 (2015).

8. Clark CM, et al. Use of florbetapir-PET for imaging beta-amyloid pathology. Jama 305, 275-283 (2011).

9. Villemagne VL, Dore V, Burnham SC, Masters CL, Rowe CC. Imaging tau and amyloid-beta proteinopathies in Alzheimer disease and other conditions. Nat Rev Neurol 14, 225-236 (2018).

10. Sabri O, et al. Florbetaben PET imaging to detect amyloid beta plaques in Alzheimer's disease: phase 3 study. Alzheimer's \& dementia : the journal of the Alzheimer's Association 11, 964-974 (2015).

11. Jack CR, Jr., et al. NIA-AA Research Framework: Toward a biological definition of Alzheimer's disease. Alzheimer's \& dementia : the journal of the Alzheimer's Association 14, 535-562 (2018).

12. Jack CR, Jr., et al. Hypothetical model of dynamic biomarkers of the Alzheimer's pathological cascade. The Lancet Neurology 9, 119-128 (2010).

13. Whitesell JD, et al. Whole brain imaging reveals distinct spatial patterns of amyloid beta deposition in three mouse models of Alzheimer's disease. The Journal of comparative neurology 527, 2122-2145 (2019).

14. Klohs J, et al. Longitudinal Assessment of Amyloid Pathology in Transgenic ArcAbeta Mice Using Multi-Parametric Magnetic Resonance Imaging. PloS one 8, e66097 (2013).

15. Bacskai BJ, et al. Four-dimensional multiphoton imaging of brain entry, amyloid binding, and clearance of an amyloid- $\beta$ ligand in transgenic mice. Proc Natl Acad Sci U S A 100, 12462-12467 (2003).

16. Hefendehl JK, et al. Long-term in vivo imaging of beta-amyloid plaque appearance and growth in a mouse model of cerebral beta-amyloidosis. The Journal of neuroscience : the official journal of the Society for Neuroscience 31, 624-629 (2011).

17. Meyer-Luehmann $\mathrm{M}$, et al. Rapid appearance and local toxicity of amyloid-beta plaques in a mouse model of Alzheimer's disease. Nature 451, 720-724 (2008). 
18. Petrova E, Yoon SJ, Pelivanov I, O'Donnell M. Photoacoustic assay for probing amyloid formation: Feasibility study. Progress in Biomedical Optics and Imaging - Proceedings of SPIE 10494, (2018).

19. Hu S, Yan P, Maslov K, Lee J-M, Wang LV. Optical-resolution photoacoustic microscopy of amyloid-beta deposits in vivo. SPIE BiOS 7564, 4 (2010).

20. Calvo-Rodriguez $\mathrm{M}$, et al. In vivo detection of tau fibrils and amyloid beta aggregates with luminescent conjugated oligothiophenes and multiphoton microscopy. Acta Neuropathol Commun 7, 171 (2019).

21. Maier FC, et al. Longitudinal PET-MRI reveals beta-amyloid deposition and rCBF dynamics and connects vascular amyloidosis to quantitative loss of perfusion. Nat Med 20, 1485-1492 (2014).

22. Rodriguez-Vieitez E, et al. Astrocytosis precedes amyloid plaque deposition in Alzheimer APPswe transgenic mouse brain: a correlative positron emission tomography and in vitro imaging study. European journal of nuclear medicine and molecular imaging 42, 1119-1132 (2015).

23. Brendel $\mathrm{M}$, et al. Impact of partial volume effect correction on cerebral beta-amyloid imaging in APP-Swe mice using [(18)F]-florbetaben PET. Neurolmage 84, 843-853 (2014).

24. Poisnel G, et al. PET imaging with [18F]AV-45 in an APP/PS1-21 murine model of amyloid plaque deposition. Neurobiol Aging 33, 2561-2571 (2012).

25. Sehlin D, Fang XT, Cato L, Antoni G, Lannfelt L, Syvanen S. Antibody-based PET imaging of amyloid beta in mouse models of Alzheimer's disease. Nature communications 7, 10759 (2016).

26. Hintersteiner $M$, et al. In vivo detection of amyloid-beta deposits by near-infrared imaging using an oxazine-derivative probe. Nat Biotechnol 23, 577-583 (2005).

27. Hyde D, et al. Hybrid FMT-CT imaging of amyloid-beta plaques in a murine Alzheimer's disease model. Neurolmage 44, 1304-1311 (2009).

28. Zhang $X$, et al. Near-infrared fluorescence molecular imaging of amyloid beta species and monitoring therapy in animal models of Alzheimer's disease. Proc Natl Acad Sci U S A 112, 97349739 (2015).

29. Razansky D, et al. Multispectral opto-acoustic tomography of deep-seated fluorescent proteins in vivo. Nature Photonics 3, 412 (2009). 
30. Omar M, Aguirre J, Ntziachristos V. Optoacoustic mesoscopy for biomedicine. Nature Biomedical Engineering 3, 354-370 (2019).

31. Ovsepian SV, Olefir I, Westmeyer G, Razansky D, Ntziachristos V. Pushing the Boundaries of Neuroimaging with Optoacoustics. Neuron 96, 966-988 (2017).

32. Luís Deán-Ben X, Razansky D. Adding fifth dimension to optoacoustic imaging: volumetric timeresolved spectrally enriched tomography. Light: Science \&Amp; Applications 3, e137 (2014).

33. Wang LV, Hu S. Photoacoustic tomography: in vivo imaging from organelles to organs. Science 335, 1458-1462 (2012).

34. Taruttis A, Ntziachristos V. Advances in real-time multispectral optoacoustic imaging and its applications. Nature Photonics 9, 219-227 (2015).

35. Dean-Ben $\mathrm{XL}$, et al. Functional optoacoustic neuro-tomography for scalable whole-brain monitoring of calcium indicators. Light Sci App/ 5, e16201 (2016).

36. Knobloch M, Konietzko U, Krebs DC, Nitsch RM. Intracellular Abeta and cognitive deficits precede beta-amyloid deposition in transgenic arcAbeta mice. Neurobiol Aging 28, 1297-1306 (2007).

37. Merlini M, Meyer EP, Ulmann-Schuler A, Nitsch RM. Vascular beta-amyloid and early astrocyte alterations impair cerebrovascular function and cerebral metabolism in transgenic arcAbeta mice. Acta neuropathologica 122, 293-311 (2011).

38. Ni R, et al. fMRI Reveals Mitigation of Cerebrovascular Dysfunction by Bradykinin Receptors 1 and 2 Inhibitor Noscapine in a Mouse Model of Cerebral Amyloidosis. Front aging neurosci 11, 27-27 (2019).

39. Ni R, Rudin M, Klohs J. Cortical hypoperfusion and reduced cerebral metabolic rate of oxygen in the arcAbeta mouse model of Alzheimer's disease. Photoacoustics 10, 38-47 (2018).

40. Klohs J, et al. Quantitative assessment of microvasculopathy in $\operatorname{arcA} \beta$ mice with USPIOenhanced gradient echo MRI. J Cereb Blood Flow Metab 36, 1614-1624 (2016).

41. Radde $\mathrm{R}$, et al. Abeta42-driven cerebral amyloidosis in transgenic mice reveals early and robust pathology. EMBO Rep 7, 940-946 (2006). 
42. Ozbek A, Deán-Ben XL, Razansky D. Realtime parallel back-projection algorithm for threedimensional optoacoustic imaging devices. SPIE (2013).

43. Ding L, Dean-Ben XL, Razansky D. Efficient 3-D Model-Based Reconstruction Scheme for Arbitrary Optoacoustic Acquisition Geometries. IEEE Trans Med Imaging 36, 1858-1867 (2017).

44. Cox B, Laufer J, Beard P, Arridge S. Quantitative spectroscopic photoacoustic imaging: a review. Journal of Biomedical Optics 17, 061202 (2012).

45. Laufer J, Jathoul A, Pule M, Beard P. In vitro characterization of genetically expressed absorbing proteins using photoacoustic spectroscopy. Biomedical Optics Express 4, 2477-2490 (2013).

46. Prahl S. https://omlc.org/spectra/hemoglobin/. (ed^(eds).

47. Lein ES, et al. Genome-wide atlas of gene expression in the adult mouse brain. Nature 445, 168176 (2007).

48. Ma Y, et al. A three-dimensional digital atlas database of the adult C57BL/6J mouse brain by magnetic resonance microscopy. Neuroscience 135, 1203-1215 (2005).

49. Chen Z, Dean-Ben XL, Gottschalk S, Razansky D. Hybrid system for in vivo epifluorescence and 4D optoacoustic imaging. Opt Lett 42, 4577-4580 (2017).

50. Chung K, et al. Structural and molecular interrogation of intact biological systems. Nature 497, 332-337 (2013).

51. Yang B, et al. Single-cell phenotyping within transparent intact tissue through whole-body clearing. Cell 158, 945-958 (2014).

52. Voigt FF, et al. The mesoSPIM initiative: open-source light-sheet microscopes for imaging cleared tissue. Nat Methods 16, 1105-1108 (2019).

53. Burton NC, et al. Multispectral Opto-acoustic Tomography (MSOT) of the Brain and Glioblastoma Characterization. Neurolmage 65, 522-528 (2013).

54. Hupple CW, Morscher S, Burton NC, Pagel MD, McNally LR, Cárdenas-Rodríguez J. A lightfluence-independent method for the quantitative analysis of dynamic contrast-enhanced multispectral optoacoustic tomography (DCE MSOT). Photoacoustics 10, 54-64 (2018). 
55. Ni R, Vaas M, Ren W, Klohs J. Non-invasive detection of acute cerebral hypoxia and subsequent matrix-metalloproteinase activity in a mouse model of cerebral ischemia using multispectraloptoacoustic-tomography. Neurophotonics 5, 015005 (2018).

56. Ren W, Skulason H, Schlegel F, Rudin M, Klohs J, Ni R. Automated registration of magnetic resonance imaging and optoacoustic tomography data for experimental studies. Neurophotonics 6, 1-10, 10 (2019).

57. Jährling N, Becker K, Wegenast-Braun BM, Grathwohl SA, Jucker M, Dodt H-U. Cerebral $\beta$ Amyloidosis in Mice Investigated by Ultramicroscopy. PloS one 10, e0125418 (2015).

58. Olsson F, et al. Characterization of intermediate steps in amyloid beta (Abeta) production under near-native conditions. J Biol Chem 289, 1540-1550 (2014).

59. Kakuda $\mathrm{N}$, et al. Distinct deposition of amyloid-beta species in brains with Alzheimer's disease pathology visualized with MALDI imaging mass spectrometry. Acta Neuropathol Commun 5, 73 (2017).

60. Fluhrer $\mathrm{R}$, et al. Identification of a beta-secretase activity, which truncates amyloid beta-peptide after its presenilin-dependent generation. J Biol Chem 278, 5531-5538 (2003).

61. Cabrera $\mathrm{E}$, et al. Abeta truncated species: Implications for brain clearance mechanisms and amyloid plaque deposition. Biochim Biophys Acta Mol Basis Dis 1864, 208-225 (2018).

62. Ni R, et al. Amyloid tracers binding sites in autosomal dominant and sporadic Alzheimer's disease. Alzheimer's \& dementia : the journal of the Alzheimer's Association 13, 419-430 (2017).

63. Lord A, et al. Observations in APP bitransgenic mice suggest that diffuse and compact plaques form via independent processes in Alzheimer's disease. The American journal of pathology 178, 2286-2298 (2011).

64. $\mathrm{Ni} \mathrm{R}$, et al. Transcranial in vivo detection of $A \beta$ at single plaque resolution with large-field multifocal illumination fluorescence microscopy. bioRxiv, 2020.2002.2001.929844 (2020).

65. Rasmussen J, et al. Amyloid polymorphisms constitute distinct clouds of conformational variants in different etiological subtypes of Alzheimer's disease. Proc Natl Acad Sci U S A 114, 1301813023 (2017). 
66. Snellman A, et al. In vivo PET imaging of beta-amyloid deposition in mouse models of Alzheimer's disease with a high specific activity PET imaging agent [(18)F]flutemetamol. EJNMMI Res 4, 37 (2014).

67. Ni R, Gillberg PG, Bergfors A, Marutle A, Nordberg A. Amyloid tracers detect multiple binding sites in Alzheimer's disease brain tissue. Brain : a journal of neurology 136, 2217-2227 (2013).

68. Kollmer $\mathrm{M}$, et al. Cryo-EM structure and polymorphism of Abeta amyloid fibrils purified from Alzheimer's brain tissue. Nature communications 10, 4760 (2019).

69. Liebmann T, Renier N, Bettayeb K, Greengard P, Tessier-Lavigne M, Flajolet M. ThreeDimensional Study of Alzheimer's Disease Hallmarks Using the iDISCO Clearing Method. Cell reports 16, 1138-1152 (2016).

70. Ni R, et al. Comparative in-vitro and in-vivo quantifications of pathological tau deposits and their association with neurodegeneration in tauopathy mouse models. Journal of nuclear medicine: official publication, Society of Nuclear Medicine 59, 960-966 (2018). 